JOURNAL OF

FUNCTION SPACES AND APPLICATIONS

Volume 5, Number 1 (2007), 1-8
(C) 2007, Scientific Horizon http://www.jfsa.net

\title{
Dual of modulation spaces
}

\section{Masaharu Kobayashi}

(Communicated by Hans Triebel)

2000 Mathematics Subject Classification. 42B35, 46A16, 46E99.

Keywords and phrases. Modulation spaces, quasi-Banach spaces, dual of modulation spaces, Inversion formula.

Abstract. The aim of this paper is the study of the dual of modulation spaces $M^{p, q}\left(\mathbf{R}^{d}\right)$ for $0<p, q<\infty$.

\section{Introduction}

We have constructed the modulation spaces $M^{p, q}\left(\mathbf{R}^{d}\right)$ in [2] for general $0<p, q \leqq \infty$, which coincide with the ususal modulation spaces when $1 \leqq p, q \leqq \infty$, and studied their basic properties. The aim of this paper is the study of the dual of $M^{p, q}\left(\mathbf{R}^{d}\right)$ for $0<p, q<\infty$. When $1 \leqq p, q<\infty$, the fact that $M^{p^{\prime}, q^{\prime}}\left(\mathbf{R}^{d}\right)$ is the dual of $M^{p, q}\left(\mathbf{R}^{d}\right)$ is already known, where $1 / p+1 / p^{\prime}=1 / q+1 / q^{\prime}=1$. (See Feichtinger [1].) So in this paper we are concerned with the dual, in particular when $p<1$ or $q<1$. Motivated by the fact that the modulation spaces have similar properties to that of the Besov spaces (Proposition 2.2), we employ H. Triebel's method [3] to study the dual. But gained results are similar to the sequence spaces $l^{p}$ rather than the Besov spaces $B_{p, q}^{s}\left(\mathbf{R}^{d}\right)$. 


\section{Preliminaries}

2.1. Basic definition. The following notations will be used throughout this article. Let $\mathcal{S}\left(\mathbf{R}^{d}\right)$ be the Schwartz space of all complex-valued rapidly decreasing infinitely differentiable functions on $\mathbf{R}^{d}$ and $\mathcal{S}^{\prime}\left(\mathbf{R}^{d}\right)$ be the topological dual of $\mathcal{S}\left(\mathbf{R}^{d}\right)$. The Fourier transform is $\hat{f}(\omega)=$ $\int f(t) e^{-2 \pi i \omega \cdot t} d t$, and the inverse Fourier transform is $\check{f}(t)=\hat{f}(-t)$. We define for $0<p<\infty$

$$
\|f\|_{L^{p}}=\left(\int_{\mathbf{R}^{d}}|f(x)|^{p} d x\right)^{\frac{1}{p}}
$$

and $\|f\|_{L^{\infty}}=$ ess. $\sup _{x \in \mathbf{R}^{d}}|f(x)|$. We use the pairing $\langle f, g\rangle$ between $f \in \mathcal{S}^{\prime}\left(\mathbf{R}^{d}\right)$ and $g \in \mathcal{S}\left(\mathbf{R}^{d}\right)$, in a manner consistent with the inner product $\langle f, g\rangle=\int f(t) \overline{g(t)} d t$ on $L^{2}\left(\mathbf{R}^{d}\right)$. For a function $f$ on $\mathbf{R}^{d}$, the translation and the modulation operators are defined by

$$
T_{x} f(t)=f(t-x), \quad \text { and } \quad M_{\omega} f(t)=e^{2 \pi i \omega \cdot t} f(t) \quad\left(x, \omega \in \mathbf{R}^{d}\right),
$$

respectively. And $\tilde{f}$ is the function defined by $\tilde{f}(x):=\overline{f(-x)}$.

2.2. Modulation spaces and basic properties. We recall the definition of the modulation spaces.

First for $\alpha>0$ we define $\Phi^{\alpha}\left(\mathbf{R}^{d}\right)$ to be the space of all $g \in \mathcal{S}\left(\mathbf{R}^{d}\right)$ satisfying

$$
\operatorname{supp} \widehat{g} \subset\{\xi|| \xi \mid \leqq 1\} \text {, and } \sum_{k \in \mathbf{Z}^{d}} \widehat{g}(\xi-\alpha k) \equiv 1, \forall \xi \in \mathbf{R}^{d} \text {. }
$$

In the following, we choose a sufficiently small $\alpha>0$ so that the function space $\Phi^{\alpha}\left(\mathbf{R}^{d}\right)$ is not empty. With this, we have defined the modulation spaces as follows:

Definition 2.1. Given a $g \in \Phi^{\alpha}\left(\mathbf{R}^{d}\right)$, and $0<p, q \leqq \infty$, we define the modulation space $M^{p, q}\left(\mathbf{R}^{d}\right)$ to be the space of all tempered distributions $f \in \mathcal{S}^{\prime}\left(\mathbf{R}^{d}\right)$ such that the quasi-norm

$$
\|f\|_{M^{p, q}}:=\left(\int_{\mathbf{R}^{d}}\left(\int_{\mathbf{R}^{d}}\left|f *\left(M_{\omega} g\right)(x)\right|^{p} d x\right)^{\frac{q}{p}} d \omega\right)^{\frac{1}{q}}
$$

is finite, with obvious modifications if $p$ or $q=\infty$.

We state basic properties of modulation spaces, which will play an important role in this article. (See [2].) 
Proposition 2.2. Let $0<p, q \leqq \infty$ and $g \in \Phi^{\alpha}\left(\mathbf{R}^{d}\right)$.Then

(a)

$$
\left(\sum_{k \in \mathbf{Z}^{d}}\left(\int_{\mathbf{R}^{d}}\left|f *\left(M_{\alpha k} g\right)(x)\right|^{p} d x\right)^{\frac{q}{p}}\right)^{\frac{1}{q}}
$$

is an equivalent quasi-norm on $M^{p, q}\left(\mathbf{R}^{d}\right)$ with modifications if $p$ or $q=\infty$.

(b) Different test functions $g_{1}, g_{2} \in \Phi^{\alpha}\left(\mathbf{R}^{d}\right)$ define the same space and equivalent quasi-norms on $M^{p, q}\left(\mathbf{R}^{d}\right)$.

(c) Let $0<p_{0} \leqq p_{1} \leqq \infty$ and $0<q_{0} \leqq q_{1} \leqq \infty$. Then

$$
M^{p_{0}, q_{0}}\left(\mathbf{R}^{d}\right) \subset M^{p_{1}, q_{1}}\left(\mathbf{R}^{d}\right) .
$$

(d) We have the continuous embeddings

$$
\mathcal{S}\left(\mathbf{R}^{d}\right) \subset M^{p, q}\left(\mathbf{R}^{d}\right) \subset \mathcal{S}^{\prime}\left(\mathbf{R}^{d}\right)
$$

for $0<p, q \leqq \infty$.

(e) $M^{p, q}\left(\mathbf{R}^{\bar{d}}\right)$ is a quasi-Banach space if $0<p, q \leqq \infty$ (Banach space if $1 \leqq p, q \leqq \infty)$.

(f) If $0<p, q<\infty$, then $\mathcal{S}\left(\mathbf{R}^{d}\right)$ is dense in $M^{p, q}\left(\mathbf{R}^{d}\right)$.

These facts have been derived from the following.

Let $0<p \leqq \infty$, and $\Gamma$ be a compact subset of $\mathbf{R}^{d}$. Then $L_{\Gamma}^{p}$ is defined by

$$
L_{\Gamma}^{p}=\left\{f \in \mathcal{S}^{\prime}\left(\mathbf{R}^{d}\right) \mid \exists \xi_{0} \in \mathbf{R}^{d}, \operatorname{supp} \widehat{f} \subset \xi_{0}+\Gamma,\|f\|_{L^{p}}<\infty\right\} .
$$

Lemma 2.3. Let $\Gamma$ be a compact subset of $\mathbf{R}^{d}$ and let $0<p \leqq q \leqq \infty$. Then there exists a positive constant $C$ ( which depends only on the diameter of $\Gamma$ and $p$ ) such that

holds for all $f \in L_{\Gamma}^{p}$.

$$
\|f\|_{L^{q}} \leqq C\|f\|_{L^{p}}
$$

Lemma 2.4. Let $0<p \leqq 1$ and $\Gamma, \Gamma^{\prime}$ be compact subsets of $\mathbf{R}^{d}$. Then there exists a positive constant $C$ (which depends only on the diameters of $\Gamma, \Gamma^{\prime}$ and $p$ ) such that

$$
\||f| *|g|\|_{L^{p}} \leqq C\|f\|_{L^{p}}\|g\|_{L^{p}}
$$

holds for all $f \in L_{\Gamma}^{p}$ and all $g \in L_{\Gamma^{\prime}}^{p}$.

In the sequel, we shall not distinguish between equivalent quasi-norms of a given quasi-normed space. 
2.3. Summary of the results. We now formulate our results.

(i) For $0<p, q \leqq 1$, we have

$$
\left(M^{p, q}\left(\mathbf{R}^{d}\right)\right)^{\prime}=M^{p^{\prime}, q^{\prime}}\left(\mathbf{R}^{d}\right)=M^{\infty, \infty}\left(\mathbf{R}^{d}\right),
$$

(ii) for $0<p<1,1<q<\infty$,

$$
M^{p^{\prime}, q^{\prime}}\left(\mathbf{R}^{d}\right) \subset\left(M^{p, q}\left(\mathbf{R}^{d}\right)\right)^{\prime} \subset M^{\infty, \infty}\left(\mathbf{R}^{d}\right),
$$

(iii) and for $1 \leqq p<\infty, 0<q<1$,

$$
\left(M^{p, q}\left(\mathbf{R}^{d}\right)\right)^{\prime}=M^{p^{\prime}, q^{\prime}}\left(\mathbf{R}^{d}\right)=M^{p^{\prime}, \infty}\left(\mathbf{R}^{d}\right) .
$$

Here the symbol $\left(M^{p, q}\left(\mathbf{R}^{d}\right)\right)^{\prime}$ is used to denote all bounded linear functionals on $M^{p, q}\left(\mathbf{R}^{d}\right)$ and $p^{\prime}$ is a conjugate exponent of $p$ which is determined in the usual way by $\frac{1}{p}+\frac{1}{p^{\prime}}=1$ if $1 \leqq p<\infty$, and we put $p^{\prime}=\infty$ if $0<p<1 . q^{\prime}$ is determined in the same way.

Suppose $0<p, q<1$. Then for the sequence spaces, $\left(l^{p}\right)^{\prime}=l^{\infty}$, that is all dual spaces are equal. But for the Besov spaces, Triebel [3] has proved that $\left(B_{p, q}^{s}\left(\mathbf{R}^{d}\right)\right)^{\prime}=B_{\infty, \infty}^{-s+d\left(\frac{1}{p}-1\right)}$. In this sense, the modulation spaces are similar to the sequence spaces rather than the Besov spaces for the dual. At present, we don't know whether or not $\left(M^{p, q}\left(\mathbf{R}^{d}\right)\right)^{\prime}=M^{p^{\prime}, q^{\prime}}\left(\mathbf{R}^{d}\right)$ when $0<p<1<q<\infty$.

2.4. Technical lemmas. Our theory deeply depends on the following transformation from a function $f$ on $\mathbf{R}^{d}$ into a family of functions on $\mathbf{R}^{d}$,

$$
f \mapsto\left\{f *\left(M_{\alpha k} g\right)(x)\right\}_{k \in \mathbf{Z}^{d}} .
$$

It is natural to ask whether it is possible to recover $f$. The following key lemma in this paper asserts that this transformation is invertible on $\mathcal{S}^{\prime}\left(\mathbf{R}^{d}\right)$ and also implies the duality on $M^{p, q}\left(\mathbf{R}^{d}\right)$.

Lemma 2.5. (The Inversion Formula) Let $f \in \mathcal{S}^{\prime}\left(\mathbf{R}^{d}\right), \psi \in \mathcal{S}\left(\mathbf{R}^{d}\right)$ and $g \in \Phi^{\alpha}\left(\mathbf{R}^{d}\right)$. Then there exists a positive constant $N$ which depends only on the size of supp $\hat{g}, \alpha>0$ and the dimension $d$, such that

$$
\langle f, \psi\rangle=\sum_{k \in \mathbf{Z}^{d}} \sum_{|r| \leqq N}\left\langle f *\left(M_{\alpha k} g\right), \psi *\left(M_{\alpha(k+r)} g\right)\right\rangle .
$$

Proof. First note that there exists a constant $N$ (depending only on the size of supp $\widehat{g}, \alpha>0$ and dimension $d)$ such that $\overline{T_{\alpha k} \widehat{g}}=\sum_{|r| \leqq N} T_{\alpha(k+r)} \widehat{g}$. 
$\overline{T_{\alpha k} \widehat{g}}$ for all $k \in \mathbf{Z}^{d}$. Since $\widehat{\psi}=\lim _{n \rightarrow \infty} \sum_{|k| \leqq n} \overline{T_{\alpha k} \widehat{g}} \cdot \widehat{\psi}$ in $\mathcal{S}\left(\mathbf{R}^{d}\right)$, we have

$$
\begin{aligned}
\langle f, \psi\rangle & =\langle\widehat{f}, \widehat{\psi}\rangle \\
& =\left\langle\widehat{f}, \lim _{n \rightarrow \infty} \sum_{|k| \leqq n} \overline{T_{\alpha k} \widehat{g}} \cdot \widehat{\psi}\right\rangle \\
& =\lim _{n \rightarrow \infty} \sum_{|k| \leqq n}\left\langle\widehat{f}, \overline{T_{\alpha k} \widehat{g}} \cdot \widehat{\psi}\right\rangle \\
& =\sum_{k \in \mathbf{Z}^{d}}\left\langle\widehat{f}, \sum_{|r| \leqq N} T_{\alpha(k+r)} \widehat{g} \cdot \overline{T_{\alpha k} \widehat{g}} \cdot \widehat{\psi}\right\rangle \\
& =\sum_{k \in \mathbf{Z}^{d}} \sum_{|r| \leqq N}\left\langle\widehat{f} \cdot T_{\alpha k} \widehat{g}, T_{\alpha(k+r)} \widehat{g} \cdot \widehat{\psi}\right\rangle \\
& =\sum_{k \in \mathbf{Z}^{d}} \sum_{|r| \leqq N}\left\langle f *\left(M_{\alpha k} g\right), \psi *\left(M_{\alpha(k+r)} g\right)\right\rangle .
\end{aligned}
$$

Lemma 2.6. Let $f \in \mathcal{S}^{\prime}\left(\mathbf{R}^{d}\right)$ and $\eta \in \mathcal{S}\left(\mathbf{R}^{d}\right)$. Then the convolution $f * \eta$ exists in $\mathcal{O}_{M}$ and can be represented in the form

$$
\langle f * \eta(x), \psi(x)\rangle=\langle f(x), \tilde{\eta} * \psi(x)\rangle, \quad \psi \in \mathcal{S}\left(\mathbf{R}^{d}\right) .
$$

Lemma 2.7. For $g \in \mathcal{S}\left(\mathbf{R}^{d}\right), k \in \mathbf{Z}^{d}, \alpha>0$ and $\xi \in \mathbf{R}^{d}$ we have

$$
\left(\overline{T_{\alpha k} \hat{g}}\right)(\xi)=\left(\widetilde{M_{\alpha k} g}\right)^{\wedge}(\xi)=\left(M_{\alpha k} \tilde{g}\right)^{\wedge}(\xi) .
$$

\section{Dual spaces}

In this section we study the space $\left(M^{p, q}\left(\mathbf{R}^{d}\right)\right)^{*}$ of bounded anti-linear functionals on $M^{p, q}\left(\mathbf{R}^{d}\right)$, equivalently, study the dual of $M^{p, q}\left(\mathbf{R}^{d}\right)$. We mean here, a functional $l$ on a quasi-Banach space $B$ is anti-linear if for any $\phi, \psi \in B$ and $\alpha \in \mathbf{C}$, we have $l(\phi+\psi)=l(\phi)+l(\psi)$ and $l(\alpha \phi)=\bar{\alpha} l(\phi)$. Throughout this section, $g$ denotes a function in $\Phi^{\alpha}\left(\mathbf{R}^{d}\right)$. If $1 \leqq p<\infty$, then the conjugate exponent $p^{\prime}$ of $p$ is determined in the usual way by $\frac{1}{p}+\frac{1}{p^{\prime}}=1$. Moreover, we put $p^{\prime}=\infty$ if $0<p<1$.

3.1. The case $1 \leqq p<\infty$.

Theorem 3.1. Let $1 \leqq p<\infty$ and $0<q \leqq 1$.

(a) Suppose $f \in M^{p^{\prime}, \infty}\left(\mathbf{R}^{d}\right)$. Then the anti-linear functional $l$ given by

$$
l(\psi)=\sum_{k \in \mathbf{Z}^{d}} \sum_{|r| \leqq N}\left\langle f *\left(M_{\alpha k} g\right), \psi *\left(M_{\alpha(k+r)} g\right)\right\rangle,
$$


initially defined on the dense subspace $\mathcal{S}\left(\mathbf{R}^{d}\right)$ of $M^{p, q}\left(\mathbf{R}^{d}\right)$, has a unique bounded extension to $M^{p, q}\left(\mathbf{R}^{d}\right)$ and satisfies

$$
\|l\|_{\left(M^{p, q}\right)^{*}} \leqq C|| f \|_{M^{p^{\prime}, \infty}} .
$$

(b) Conversely, every bounded anti-linear functional $l$ on $M^{p, q}\left(\mathbf{R}^{d}\right)$ can be realized as above, with an $f \in M^{p^{\prime}, \infty}\left(\mathbf{R}^{d}\right)$, and with

$$
\|f\|_{M^{p^{\prime}, \infty}} \leqq C^{\prime}|| l \|_{\left(M^{p, q}\right)^{*}} .
$$

Proof. (a) Let $f \in M^{p^{\prime}, \infty}\left(\mathbf{R}^{d}\right), \psi \in \mathcal{S}\left(\mathbf{R}^{d}\right)$. Then by Hölder's inequality and continuous embedding for sequence spaces, $l^{q} \subset l^{1}$ if $q \leqq 1$, we have

$$
\begin{aligned}
|l(\psi)| & \leqq \sum_{|r| \leqq N} \sum_{k \in \mathbf{Z}^{d}}\left\|f *\left(M_{\alpha k} g\right)(x)\right\|_{L^{p^{\prime}}}\left\|\psi *\left(M_{\alpha(k+r)} g\right)(x)\right\|_{L^{p}} \\
& \leqq C\|\| f *\left(M_{\alpha k} g\right)\left\|_{L^{p^{\prime}}}\right\|_{l^{\infty}}\|\| \psi *\left(M_{\alpha k} g\right)\left\|_{L_{p}}\right\|_{l^{q}} \\
& =C\|f\|_{M^{p^{\prime}, \infty}}\|\psi\|_{M^{p, q}}
\end{aligned}
$$

Thus $l(\psi)$ defined by (1) converges and defines a bounded anti-linear functional $l$ on $\mathcal{S}\left(\mathbf{R}^{d}\right)$ in the quasi-norm in $M^{p, q}\left(\mathbf{R}^{d}\right)$. Then since $\mathcal{S}\left(\mathbf{R}^{d}\right)$ is dense in $M^{p, q}\left(\mathbf{R}^{d}\right)$ and $M^{p, q}\left(\mathbf{R}^{d}\right)$ is a quasi-Banach space, we have desired result.

(b) We first note that $\left(M^{p, q}\left(\mathbf{R}^{d}\right)\right)^{*}$ is embedded in $\mathcal{S}^{\prime}\left(\mathbf{R}^{d}\right)$, since $\mathcal{S}\left(\mathbf{R}^{d}\right)$ is a dense subset of $M^{p, q}\left(\mathbf{R}^{d}\right)$. Therefore, setting $\langle l, \psi\rangle:=l(\psi)$, we obtain (1) with $f=l$ by virtue of Lemma 2.5 .

Let $1 \leqq p<\infty, 0<q \leqq 1$ and $l \in\left(M^{p, q}\left(\mathbf{R}^{d}\right)\right)^{*}$. We show that for each $k \in \mathbf{Z}^{d}, l *\left(M_{\alpha k} g\right)(x)$ is a well-defined element of $L^{p^{\prime}}\left(\mathbf{R}^{d}\right)$. With $\psi \in \mathcal{S}\left(\mathbf{R}^{d}\right)$, we estimate

$$
\left|\left\langle l *\left(M_{\alpha k} g\right), \psi\right\rangle\right|=\left|\left\langle l,\left(\widetilde{M_{\alpha k} g}\right) * \psi\right\rangle\right| \leqq\|l\|_{\left(M^{p, q}\right)^{*}} \mid\left(\widetilde{M_{\alpha k} g}\right) * \psi \|_{M^{p, q}} .
$$

By Lemma 2.7, the following relation holds true:

$\operatorname{supp}\left(\widetilde{M_{\alpha k} g}\right)^{\wedge} \cap \bigcup_{n \in \mathbf{Z}^{d}} \operatorname{supp}\left(M_{\alpha n} g\right)^{\wedge}=\bigcup_{|r| \leqq N} \operatorname{supp}\left(\widetilde{M_{\alpha k} g}\right)^{\wedge} \cap \operatorname{supp}\left(M_{\alpha(k+r)} g\right)^{\wedge}$. 
From this and Young's inequality, we have

$$
\begin{aligned}
\left\|\left(\widetilde{M_{\alpha k} g}\right) * \psi\right\|_{M^{p, q}}^{q} & =\sum_{n \in \mathbf{Z}^{d}}\left(\int_{\mathbf{R}^{d}}\left|\left(\widetilde{M_{\alpha k} g}\right) * \psi * M_{\alpha n} g(x)\right|^{p} d x\right)^{\frac{q}{p}} \\
& =\sum_{|r| \leqq N}\left(\int_{\mathbf{R}^{d}}\left|\left(\widetilde{M_{\alpha k} g}\right) * \psi * M_{\alpha(k+r)} g(x)\right|^{p} d x\right)^{\frac{q}{p}} \\
& \leqq\|\psi\|_{L^{p}}^{q} \sum_{|r| \leqq N}\left\|\left(M_{\alpha k} \tilde{g}\right) *\left(M_{\alpha(k+r)} g\right)\right\|_{L^{1}}^{q}=C\|\psi\|_{L^{p}}^{q} .
\end{aligned}
$$

Thus for each $k \in \mathbf{Z}^{d}, l *\left(M_{\alpha k} g\right)(x)$ is a well-defined element of $L^{p^{\prime}}\left(\mathbf{R}^{d}\right)$. Taking supremum over $k \in \mathbf{Z}^{d}$, we obtain the estimate

$$
\left\|l||_{M^{p^{\prime}, q^{\prime}}}=\sup _{k \in \mathbf{Z}^{d}}\right\| l *\left(M_{\alpha k} g\right) \|_{L^{p^{\prime}}}<\infty .
$$

\subsection{The case $0<p<1$.}

Theorem 3.2. Let $0<p<1$ and $0<q<\infty$.

(a) Suppose $f \in M^{\infty, q^{\prime}}\left(\mathbf{R}^{d}\right)$. Then the anti-linear functional $l$ given by

$$
l(\psi)=\sum_{k \in \mathbf{Z}^{d}} \sum_{|r| \leqq N}\left\langle f *\left(M_{\alpha k} g\right), \psi *\left(M_{\alpha(k+r)} g\right)\right\rangle,
$$

initially defined on the dense subspace $\mathcal{S}\left(\mathbf{R}^{d}\right)$ of $M^{p, q}\left(\mathbf{R}^{d}\right)$, has a unique bounded extension to $M^{p, q}\left(\mathbf{R}^{d}\right)$ and satisfies

$$
\|l\|_{\left(M^{p, q}\right)^{*}} \leqq C|| f \|_{M^{p^{\prime}, \infty}} .
$$

(b) Every bounded anti-linear functional $l$ on $M^{p, q}\left(\mathbf{R}^{d}\right)$ can be realized as above, with an $f \in M^{\infty, \infty}\left(\mathbf{R}^{d}\right)$, and with

$$
\|f\|_{M^{\infty, \infty}} \leqq C^{\prime}|| l \|_{\left(M^{p, q}\right)^{*}}
$$

Proof. (a). Let $f \in M^{\infty, q^{\prime}}\left(\mathbf{R}^{d}\right)$. Then using Lemma 2.3, we have

$$
\begin{aligned}
|l(\psi)| & \leqq \sum_{k \in \mathbf{Z}^{d}} \sum_{|r| \leqq N}\left|\left\langle f *\left(M_{\alpha k} g\right), \psi *\left(M_{\alpha(k+r)} g\right)\right\rangle\right| \\
& \leqq \sum_{k \in \mathbf{Z}^{d}} \sum_{|r| \leqq N}\left\|f *\left(M_{\alpha k} g\right)(x)\right\|_{L^{\infty}}\left\|\psi *\left(M_{\alpha(k+r)} g\right)(x)\right\|_{L^{1}} \\
& \leqq c \sum_{|r| \leqq N} \sum_{k \in \mathbf{Z}^{d}}\left\|f *\left(M_{\alpha k} g\right)(x)\right\|_{L^{\infty}}\left\|\psi *\left(M_{\alpha(k+r)} g\right)(x)\right\|_{L^{p}}
\end{aligned}
$$




$$
\leqq C\|f\|_{M^{\infty, q^{\prime}}}\|\psi\|_{M^{p, q}} .
$$

In the last inequality, we use Hölder's inequality with $k \in \mathbf{Z}^{d}$ if $q \geqq 1$, and continuous embedding $l^{q} \subset l^{1}$ if $q \leqq 1$.

(b). Let $l \in\left(M^{p, q}\left(\mathbf{R}^{d}\right)\right)^{*}$. Then using the definition of a convolution, we have

(3) $\left|l *\left(M_{\alpha k} g\right)\left(x_{0}\right)\right|=\left|\left\langle l, \overline{M_{\alpha k} g\left(x_{0}-\cdot\right)}\right\rangle\right| \leqq\left. c|| l\right|_{\left(M^{p, q}\right)^{*}}|| M_{\alpha k} g\left(x_{0}-\cdot\right) \|\left.\right|_{M^{p, q}}$.

holds for all $x_{0} \in \mathbf{R}^{d}$ and $k \in \mathbf{Z}^{d}$. Moreover,

$$
\begin{aligned}
\left\|M_{\alpha k} g\left(x_{0}-\cdot\right)\right\|_{M^{p, q}}^{q} & =\sum_{n \in \mathbf{Z}^{d}}\left(\int\left|\left(M_{\alpha k}\left(x_{0}-\cdot\right)\right) *\left(M_{\alpha n} g\right)(x)\right|^{p} d x\right)^{\frac{q}{p}} \\
& =\sum_{|r| \leqq N}\left\|M_{\alpha k} g\left(x_{0}-\cdot\right) * M_{\alpha(k+r)} g(x)\right\|_{L^{p}}^{q} .
\end{aligned}
$$

It follows from by Lemma 2.4 that the last sum is bounded by

$$
C \sum_{|r| \leqq N}\left\|M_{\alpha k} g\left(x_{0}-\cdot\right)\right\|_{L^{p}}^{q}\left\|M_{\alpha(k+r)} g(x)\right\|_{L^{p}}^{q}
$$

where $C$ is independent of $x_{0}$ and $k$. Combining this estimate with (3) and taking supremum over $x_{0} \in \mathbf{R}^{d}$ and $k \in \mathbf{Z}^{d}$, we get the desired result.

\section{References}

[1] H. G. Feichtinger, A new class of function spaces, in Proc.Conf. "Constructive Function Theory", Kiew 1983, (1984).

[2] M. Kobayashi, Modulation spaces $M^{p, q}$ for $0<p, q \leqq \infty$, J. Function Spaces Appl., 4(3) (2006), 329-341.

[3] H. Triebel, Theory of Function Spaces, Birkhäuser, Boston, 1983.

Department of Mathematics

Tokyo University of Science

Kagurazaka 1-3

Shinjuku-ku

Tokyo 162-8601

Japan

(E-mail : kobayashi@jan.rikadai.jp) 


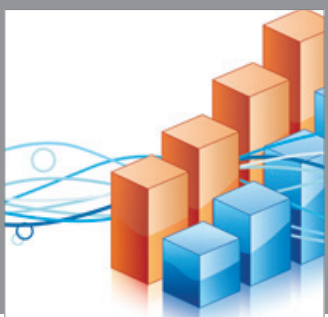

Advances in

Operations Research

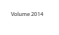

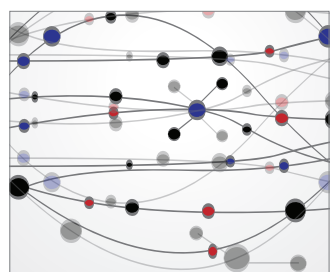

\section{The Scientific} World Journal
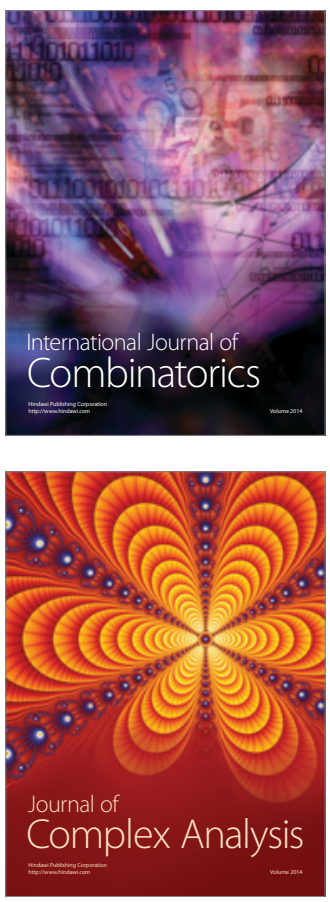

International Journal of

Mathematics and

Mathematical

Sciences
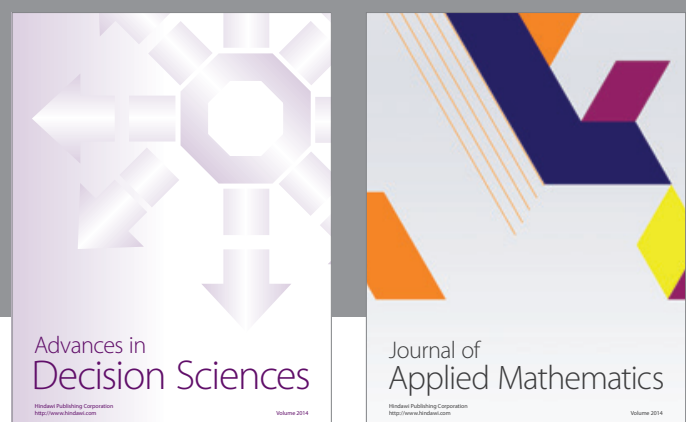

Journal of

Applied Mathematics
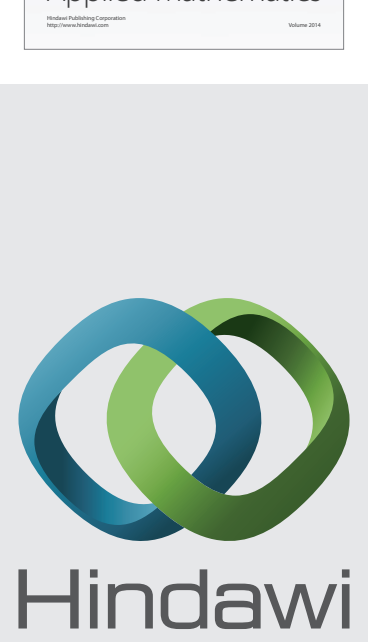

Submit your manuscripts at http://www.hindawi.com
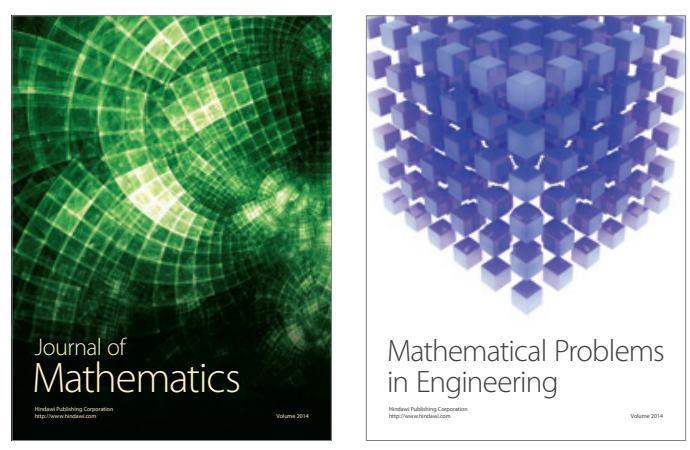

Mathematical Problems in Engineering
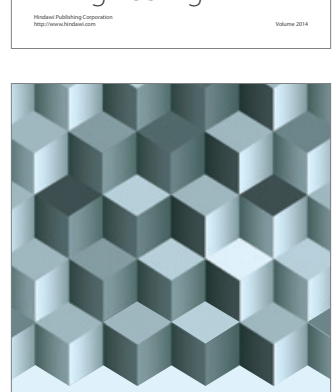

Journal of

Function Spaces
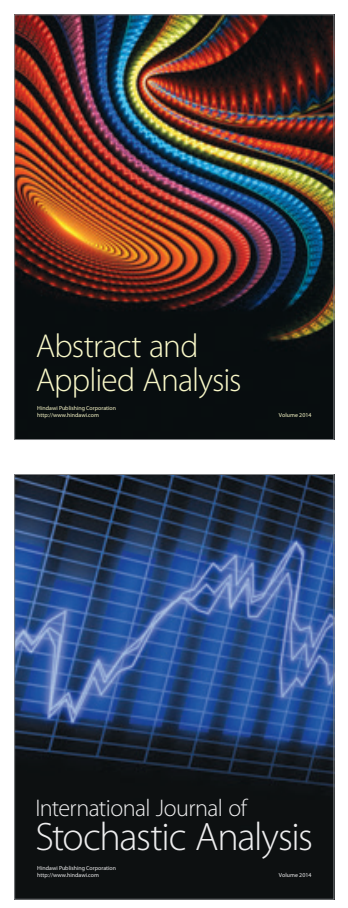

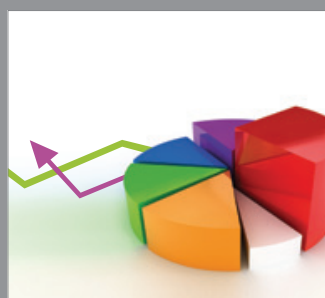

ournal of

Probability and Statistics

Promensencen
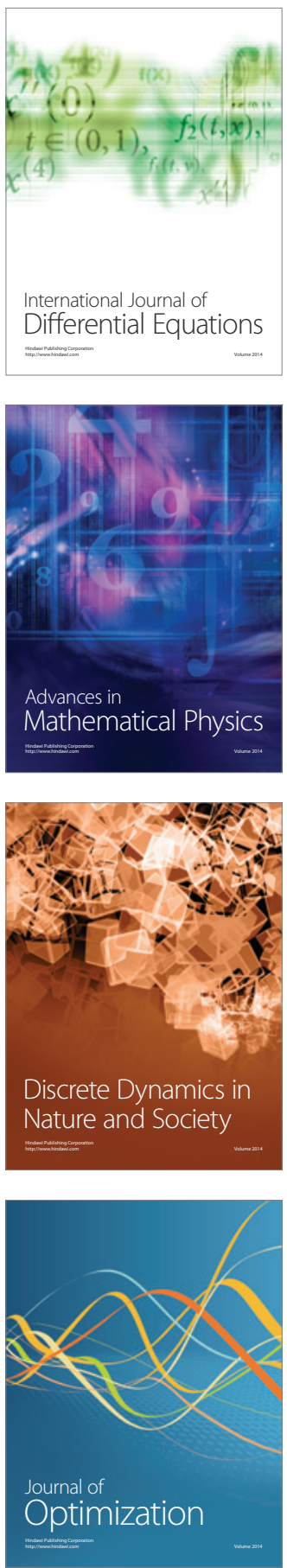